Under the new policy, BCG vaccination will be offered to infants in communities with an average incidence of tuberculosis of at least 40 per 100000 and to unvaccinated individuals who come from, or whose parents or grandparents come from, countries where the incidence exceeds 40 per 100000 . Most people born in the United Kingdom will thus probably never receive $B C G$ vaccination, and most will not be exposed to mycobacteria. This means that tuberculin testing will become increasingly efficient as a means of identifying people exposed to and latently infected with the tubercle bacillus, who may be given prophylaxis.

The change from routine to targeted vaccination is accompanied by technical changes. The Glaxo BCG vaccine has been replaced by one from the Danish Statens Seruminstitut and the multipuncture "Heaf" technique for tuberculin testing is being replaced by the intradermal injection "Mantoux" technique, which is the standard in the rest of the world. All of these changes bring the UK's approach to preventing infection with tuberculosis in line with policies and practice in many other countries.

BCG vaccination will continue to have an important role in protecting children in high risk populations from tuberculosis. Coupled with vigorous efforts to identify and appropriately treat cases, and to ascertain and offer prophylaxis to people with latent infection, the new policy should allow more efficient control of tuberculosis in the entire UK population.

Paul Fine professor of communicable disease epidemiology

Department of Infectious and Topical Diseases, London School of Hygiene and Tropical Medicine, London WC1E 7HT (paul.fine@lshtm.ac.uk)

Competing interests: PF is a member of the BCG Subcommittee of the Joint Committee on Vaccination and Immunisation and took part in discussions leading to this policy change.

1 Donaldson L, Beasley C, Smith J. Changes to the BCG vaccination programme. 6 July 2005. (CMO letter.) www.immunisation.nhs.uk/files/ CMO060705.pdf (accessed 15 Sep 2005)

2 Hart PD'A, Sutherland I. BCG and vole bacillus vaccines in the prevention of tuberculosis in adolescents and early life. Final report to the Medical Research Council. BMJ 1977;ii:293-5.

3 Comstock GW, Palmer CE. Long term results of BCG vaccination in the southern United States. Am Rev Respir Dis 1966;93:171-83.

4 Fine PEM, Carneiro IAM, Milstein JB, Clements CJ Issues relating to the use of BCG in immunization programmes: a discussion document. use of BCG in immunization programmes: a discussion document. vaccine_research/documents/en/bcg_vaccines.pdf (accessed 15 Sep

5 Sutherland I, Springett VH. Effectiveness of BCG vaccination in England and Wales in 1983. Tubercle 1987;68:81-92.

6 Health Protection Agency. Tuberculosis. www.hpa.org.uk/infections/ topics_az/tb/menu.htm (accessed 15 Sep 2005).

7 International Union against Tuberculosis and Lung Disease. Criteria for discontinuation of vaccination programmes using Bacille Calmette Guerin (BCG) in countries with a low prevalence of tuberculosis. Tubercle and Lung Dis 1994;75:179-81.

\title{
The Japanese healthcare system
}

\section{The issue is to solve the "tragedy of the commons"without making another}

$\mathrm{T}$ The Japanese medical insurance system has a unique combination of characteristics that has led to the overuse of tests and drugs, unconstrained demand from patients, and an explosion of costs. Unless the system of medical insurance and reimbursement of healthcare providers changes, the combination of increasing technological advances, an ageing population, and unconstrained demand will produce a crisis in Japanese health care. Japan is only belatedly waking up to this crisis.

The Japanese medical insurance system has four characteristics that lie at the root of the problem. Firstly, Japanese citizens are covered comprehensively and exclusively by either national medical insurance (for the self employed) or social insurance (for employees). Beneficiaries have to make some co-payments, which are capped depending on income. ${ }^{1}$ Secondly, mixed private and insurance payments are prohibited-that is, beneficiaries cannot pay privately for medical services that are covered by their medical insurance. Thirdly, beneficiaries have guaranteed access to any healthcare providers, from general practitioners to specialists, without being charged a premium fee. Finally, healthcare providers and institutions are reimbursed through fees for service.

Fuelled by economic growth after the second world war and facilitated by the healthcare system, Japan has become one of the most medically advanced nations in the world, especially in its service quantity. Compared with other developed countries in the Organisation for Economic Cooperation and Development (OECD),
Japan is the runaway leader in the number of magnetic resonance imaging and computed tomography scanners per head of population. ${ }^{2}$ Because they are paid for each prescription or test rather than time spent with patients, healthcare providers, both private and public, are driven to prescribe more drugs and to order more imaging and tests.

Japanese patients visit outpatient clinics more often and stay in hospitals longer than patients in other OECD countries. ${ }^{2}$ Profits gained from a "three-hour wait, three-minute contact" consultation (with an emphasis on ordering tests and prescribing drugs during the three minutes) primarily benefit pharmaceutical and medical equipment companies. Healthcare expenditures, both per head and as a percentage of gross domestic product, continue to increase despite the economic growth rate remaining low throughout the past 10 years. In Japan's ageing society, the economic burden rests with the insurers, who ultimately raise their funds from the working population and their employers.

Japanese health care is therefore a typical case of the "tragedy of the commons." The name relates to grazing land: free access to common grazing land drives each herdsman to maximise his own take from the commons, even when it becomes overcrowded with grazing animals. Ultimately this behaviour ruins the common land, as well as those who depend on it for survival. In the Japanese system patients are the herdsmen, and specialists, medical resources, and health insurance coverage comprise the commons. A more 
cynical view holds that doctors and pharmaceutical and medical equipment companies are the herdsmen while patients and health insurance reimbursement comprise the commons. What can be done to avoid ruin? A variety of different players have made proposals for reform

In December 2002, the council of advisers to the Cabinet Office, composed of business leaders and academic economists, recommended that the ban on mixed payments should be abolished. Private payments should be allowed for any medical services not covered by medical insurance at any medical institution which fulfilled certain conditions. ${ }^{45}$ The council argued that the ban deprived Japanese patients of the chance to receive a higher or more advanced level of medical services. It also deprived the Japanese medical industry of chances to market its new technologies and drugs, thus impeding its international competitiveness. Indeed, the big three university hospitals, well known for their research activity, as well as the Japan Surgical Society expressed their agreement with this proposal.

The Japan Medical Association, commonly regarded as an interest group for private office based practitioners, campaigned against the proposal, claiming that it would deprive people with low incomes of necessary medical services. The Ministry of Health, Labour, and Welfare opposed the recommendation on the same grounds, claiming also that patient safety would be at risk if new medical technologies and drugs were used prematurely.

Last December Prime Minister Junichiro Koizumi agreed not to adopt the council's recommendation, but instead decided to expand the existing exceptional approvals system for highly advanced medical technologies. ${ }^{6}$ Under this system private payments are expected to be allowed for selected medical technologies that are not covered by medical insurance at any hospitals that fulfil certain conditions (some 2000). For new drugs that have not yet been approved, especially those approved in other developed countries, measures will be taken to ensure steady implementation of bridging short term and long term clinical trials. But no one believes that the business leaders, who have a mission to vitalise the Japanese economy, have given up their objective.

The Ministry of Health, Labour, and Welfare is currently making changes to the healthcare system. In a scheme that started in 2003 with 82 hospitals providing advanced treatments, an increasing number of acute hospitals have adopted a system of reimbursement for inpatient care based on diagnosis-procedure combinations (DPC). ${ }^{7}$ Hospitals are paid daily fees proportionate to the length of stay for each condition and treatment, irrespective of actual interventions. ${ }^{8}$ Therefore, this system gives an incentive to healthcare institutions to provide a better service in a shorter period while ordering fewer tests and prescribing fewer drugs.

The ministry is also promoting protocol based medicine. It has provided support for the development of evidence based clinical practice guidelines by academic medical societies since 1999. ${ }^{9}$ The dissemination and implementation of these guidelines is expected to improve the quality of medical care and drive the distribution of limited resources to effective treatments. But, as in other developed countries, this remains a challenging task. The government's attempts have often been challenged by the Japan Medical Association, on the grounds of "professional autonomy." But the association is currently renewing its stance on professional autonomy, ${ }^{10}$ to a positive, self regulated commitment to patient welfare based on sound clinical evidence and expertise. ${ }^{11}$

The problem is that neither the new reimbursement system nor protocol based medicine will change patients' behaviour as "herdsmen." Previously patients and physicians were driven in the same direction: more tests, more drugs. The new reimbursement system drives only physicians in the opposite direction. Indeed, conflict between patients and physicians could cause a separate tragedy. To encourage shared decision making between patients and doctors based on sound clinical evidence, including an understanding of the need to avoid unnecessary tests and drugs, the ministry has set up a task force to investigate the possibility of patients participating in the development, dissemination, and implementation of clinical practice guidelines. This task force is also working on strategies to popularise the concept of patient-physician partnership.

To date, no countermeasures for the tragedy of the commons have been found other than restricting free access to the commons. If effective actions are not soon taken to change the behaviour of both patients and healthcare providers, some restriction on Japan's free access to health care will become inevitable. Withdrawing the ban on mixed payments-and allowing patients to pay privately for extra treatments-is equivalent to giving up an important part of the commons, and could cause the worst tragedy for patients.

\section{Hideki Nomura associate professor}

Department of General Medicine, Kanazawa University Hospital, 13-1 Takara-machi, Kanazawa, Ishikawa 920-8641, Japan

(hnomura@med.kanazawa-u.ac.jp)

Takeo Nakayama associate professor

Department of Health Informatics, Kyoto University School of Public Health, Yoshida-Konoe-cho, Kyoto 606-8501, Japan

Competing interests: TN is the chair and $\mathrm{HN}$ is a member of the task force on the improvement of the development, usage, and dissemination of evidence based practice guidelines with special attention to patient and carer involvement.

Fukawa T. Public health insurance in Japan. Washington, DC: World Bank Institute, 2002

2 Organization for Economic Cooperation and Development. OECD health data 2002. Paris: OECD, 2002.

3 Hardin G. The tragedy of the commons. Science 1968;162:1243-8.

4 Council for Regulatory Reform. Second report regarding promotion of regulatory reform - Priority regulatory reform measures to promote economic vitalization. 12 Dec 2002. http://www8.cao.go.jp/kisei/en/021212report/

5 Council on Economic and Fiscal Policy. Minutes of the 28th (November 15, 2004) and the 32nd (December 8, 2004) meetings [in Japanese] www.keizai-shimon.go.jp/minutes/2004/

6 Minister of Health, Labor and Welfare. Press release on the issue of mixed payment. 15 Dec, 2004 www.mhlw.go.jp/houdou/2004/12/h1216-1.html payment. 15 Dec,
[in Japanese].

7 Official Gazette of the Japanese Government. No 3838 and 3880,23 April and 28 June 2004 [in Japanese]

Ishikawa K, Yamamoto M, Kishi DT, Nabeshima T. New prospective payment system in Japan. Am J Health-Syst Pharm 2005;62:1617-9.

9 Nakayama T, Budgell B, Tsutani K. Confusion about the clinical practice guidelines in Japan: on the way to a social consensus. Int J Qual Health Care 2003;15:359-60.

10 Tsutani K and Nagasawa M. Professional autonomy: A new perspective for relating with clinical practice guidelines. Japan Med Assoc J 2004; 47:143-9.

11 Japan Medical Association. Toward the improvement in the quality of medical service. Tokyo:JMA, 2004 [in Japanese]. 